\title{
Artificial Intelligence in Nuclear Cardiology
}

\author{
Javier Gomez ${ }^{1}$ and Rami Doukky ${ }^{1,2}$ \\ ${ }^{I}$ Division of Cardiology, Cook County Health, Chicago, Illinois; and ${ }^{2}$ Division of Cardiology, Rush University Medical Center, \\ Chicago, Illinois
}

What we want is a machine that can learn from experience. _-Alan Turing, London, 1947

$\mathbf{T}$ has been the holy grail of cardiovascular medicine. Thanks to a large body of supportive evidence, radionuclide myocardial perfusion imaging (MPI) has been established as a mainstay in the diagnostic and prognostic assessment of patients with known or suspected coronary artery disease (1). However, deriving a patientspecific risk prediction is rather difficult, as the pretest likelihood of disease and a large number of clinical, electrocardiographic, stress, perfusion, and functional data elements influence the prognostic predictive value of stress MPI (2-9). Thus, it is impossible for the human brain to integrate dozens of variables to come up with a precise predicted probability of an outcome. Even regression equation modeling has significant limitations in this domain. In the quest for perfecting risk prediction in nuclear cardiology, artificial intelligence (AI) technologies, particularly machine learning (ML), have emerged as a potential tool to improve risk prediction (10-12). In principle, ML techniques consume very large training data to generate and validate algorithms that produce continuous or binary predictions of a given diagnosis or outcome (13).

In a recent multicenter study of 2,619 patients who underwent exercise or pharmacologic SPECT/MPI using cadmium zinc telluride cameras, Betancur et al. evaluated the ability of an ML algorithm to predict major adverse cardiac events, defined as allcause mortality, nonfatal myocardial infarction, unstable angina, or late coronary revascularization, during $3 \mathrm{y}$ of follow-up. Major adverse cardiac event prediction was compared among ML with all available data (ML-combined), ML with only imaging data (ML-imaging), physician interpretation, and automated quantitative imaging analysis using stress total perfusion deficit (TPD) and ischemic TPD. The ML algorithm involved automated variable selection by ranking the information gain from each variable and modeling them using a boosted ensemble algorithm. A 10-fold stratified cross-validation was performed, such that patients were divided into 10 groups; in each cross-validation cycle, 9 groups were used for training and the 10th group was used for testing. In the process, variable selection and iterative model building are performed and evaluated in the testing subsets. Of 70 variables considered (25 imaging, 17 stress test, 28 clinical), 47 variables

Received Jan. 3, 2019; revision accepted Mar. 18, 2019.

For correspondence or reprints contact: Rami Doukky, Cook County Health, Division of Cardiology, 1901 W. Harrison St., Chicago, IL 60612. E-mail: rdoukky@cookcountyhhs.org

Published online Mar. 29, 2019.

COPYRIGHT (c) 2019 by the Society of Nuclear Medicine and Molecular Imaging. DOI: 10.2967/jnumed.118.222356 were selected (22 imaging, 8 stress test, 17 clinical) on the basis of an information gain ratio higher than 0 . The study showed that ML-combined provided a significant improvement in predictive accuracy for major adverse cardiac events when compared with expert readers, automated quantitative analysis using stress TPD, and ischemic TPD. Furthermore, ML-combined predicted major adverse cardiac events more accurately than ML-imaging (14). The study was the first to report improved prognostic accuracy using ML algorithms applied specifically to nuclear cardiac imaging. The concept of incorporating clinical, stress, and imaging parameters in complex modeling to predict outcome is not novel. Multivariate regression modeling has been attempted to incorporate clinical and imaging variables to improve risk prediction (15). However, conventional Cox or logistic multivariate regression modeling has important limitations, particularly colinearity and overfitting. ML techniques do not suffer from these particular limitations. Moreover, it is feasible for risk prediction with ML to improve on an ongoing basis as more data and outcomes are fed into the ML algorithm, so it can continuously perfect its future predictions. On the other hand, the inability of current ML techniques to incorporate time-to-event into model building may have a significant impact on the clinical implications of risk prediction. Although ML may be, in theory, superior to predictive regression equation modeling, this advantage has to be demonstrated clinically.

Beyond ML, additional AI modalities, such as deep learning (DL) technologies, have also gained great interest as a tool to analyze medical images. DL methods, using convolutional neural networks, connect directly to image pixels and learn, in a selftaught manner, imaging data in their original DICOM format $(16,17)$. To put it into context, ML can process numeric TPD values provided by quantitative MPI analysis whereas DL can analyze the image pattern itself, resembling the human eye and brain. In a multicenter cohort of 1,638 subjects, Betancur et al. also studied DL image interpretation in patients who underwent MPI followed by invasive coronary angiography within 6 mo. The authors compared quantitative TPD against a DL algorithm using a convolutional neural network architecture analyzing SPECT/ MPI polar maps. DL image analysis provided a statistically significant improvement in per-vessel as well as per-patient sensitivity in detection of obstructive coronary artery disease compared with quantitative TPD (79.8\% to $82.3 \%$ and $64.4 \%$ to $69.8 \%$, respectively), indicating that the DL approach using convolutional neural networks was superior to standard TPD (10). Combining DL image interpretation with ML data integration has the potential to further improve risk prediction. 
These 2 studies lay the foundation for the application of ML in nuclear cardiology. Despite the great promise, the full adoption of AI in clinical practice is fraught with significant challenges. First, ML algorithms require large amounts of data for training and validation. The use of such large data sets is complicated by regulatory considerations, as data ownership is not always clear. Second, data standardization for ML is challenging. Pooling data from different centers for this purpose is not simple, as different centers use disparate nomenclature, protocols, equipment, and software tools. For the same reason, the wide-scale clinical application of ML tools is not straightforward. Finally, there are significant medicolegal concerns. Although current data suggest that $\mathrm{AI}$ can be at least as accurate as expert clinicians, erroneous interpretation can still lead to a poor outcome, making it difficult to establish responsibility. Thus, for the foreseeable future, the overseeing physician will ultimately be accountable for the final product.

It is clear that we are at the gates of the next paradigm shift in medical imaging. Understandably, the rapid evolution of AI technologies may be perceived as a threat to the imaging community. Although recent data suggest that AI image interpretation and risk prediction are feasible, unsupervised AI application in routine clinical practice is not quite ready for prime-time. Despite the anxiety, much of what AI can offer should be embraced by physicians. Why should we not take every advantage in sharpening our diagnostic and prognostic acumen? Who would not benefit from the extra pair of eyes and the crystal ball of ML algorithms? For the foreseeable future, the upcoming AI developments will not replace the role of physicians but will rather support and transform it.

\section{DISCLOSURE}

No potential conflict of interest relevant to this article was reported.

\section{REFERENCES}

1. Doukky R, Hayes K, Frogge N, et al. Impact of appropriate use on the prognostic value of single-photon emission computed tomography myocardial perfusion imaging. Circulation. 2013;128:1634-1643.
2. Golzar Y, Olusanya A, Pe N, et al. The significance of automatically measured transient ischemic dilation in identifying severe and extensive coronary artery disease in regadenoson, single-isotope technetium-99m myocardial perfusion SPECT. J Nucl Cardiol. 2015;22:526-534.

3. AlJaroudi W, Campagnoli T, Fughhi I, Wassouf M, Ali A, Doukky R. Prognostic value of heart rate response during regadenoson stress myocardial perfusion imaging in patients with end stage renal disease. J Nucl Cardiol. 2016;23:560-569.

4. Poulin MF, Alexander S, Doukky R. Prognostic implications of stress modality on mortality risk and cause of death in patients undergoing office-based SPECT myocardial perfusion imaging. J Nucl Cardiol. 2016;23:202-211.

5. Doukky R, Fughhi I, Campagnoli T, Wassouf M, Ali A. The prognostic value of regadenoson SPECT myocardial perfusion imaging in patients with end-stage renal disease. J Nucl Cardiol. 2017;24:112-118.

6. Bangalore S. Stress testing in patients with chronic kidney disease: the need for ancillary markers for effective risk stratification and prognosis. $\mathrm{J} \mathrm{Nucl} \mathrm{Cardiol.}$ 2016;23:570-574.

7. Smith L, Myc L, Watson D, Beller GA, Bourque JM. A high exercise workload of $\geq 10$ METs predicts a very low risk of significant ischemia and cardiac events in older adults. J Nucl Cardiol. July 26, 2018 [Epub ahead of print].

8. Doukky R, Frogge N, Bayissa YA, et al. The prognostic value of transient ischemic dilatation with otherwise normal SPECT myocardial perfusion imaging: a cautionary note in patients with diabetes and coronary artery disease. J Nucl Cardiol. 2013;20:774-784.

9. Doukky R, Frogge N, Balakrishnan G, et al. The prognostic value of cardiac SPECT performed at the primary care physician's office. J Nucl Cardiol. 2013; 20:519-528.

10. Betancur J, Commandeur F, Motlagh M, et al. Deep learning for prediction of obstructive disease from fast myocardial perfusion SPECT: a multicenter study. JACC Cardiovasc Imaging. 2018;11:1654-1663.

11. Motwani M, Dey D, Berman DS, et al. Machine learning for prediction of allcause mortality in patients with suspected coronary artery disease: a 5-year multicentre prospective registry analysis. Eur Heart J. 2017;38:500-507.

12. Haro Alonso D, Wernick MN, Yang Y, Germano G, Berman DS, Slomka P. Prediction of cardiac death after adenosine myocardial perfusion SPECT based on machine learning. J Nucl Cardiol. March 14, 2018 [Epub ahead of print].

13. Gomez J, Doukky R, Germano G, Slomka P. New trends in quantitative nuclear cardiology methods. Curr Cardiovasc Imaging Rep. 2018;11:1.

14. Betancur J, Otaki Y, Motwani M, et al. Prognostic value of combined clinical and myocardial perfusion imaging data using machine learning. JACC Cardiovasc Imaging. 2018;11:1000-1009.

15. Ahlberg AW, Baghdasarian SB, Athar H, et al. Symptom-limited exercise combined with dipyridamole stress: prognostic value in assessment of known or suspected coronary artery disease by use of gated SPECT imaging. $\mathrm{J} \mathrm{Nucl}$ Cardiol. 2008; 15:42-56.

16. LeCun Y, Bengio Y, Hinton G. Deep learning. Nature. 2015;521:436-444.

17. Krittanawong C, Zhang H, Wang Z, Aydar M, Kitai T. Artificial intelligence in precision cardiovascular medicine. J Am Coll Cardiol. 2017;69:2657-2664. 